PNL-SA- -19633

DE92 002153

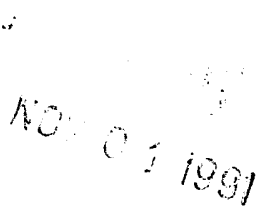

\title{
THE USER ORIENTED EVALUATION PROCESS: A PROCESS FOR PRESERVING USER NEEDS DURING INTERATIVE SYSTEM TEST AND EVALUATION
}

S. T. Hunt

A. Schur

September 1991

Presented at the Human Factors Society Annual Meeting September 2-6, 1991

San Fransisco, California

Work supported by the U.S. Department of Energy under Contract DE-AC06-76RLO 1830

Pacific Northwest Laboratory Richland, Washington 99352

DISTR:L'_TION OF THIS DOCUMENT IS UNLIMITED

\footnotetext{
This report was prepared as an account of work sponsored by an agency of the United States employees, makes any warranty, States Government nor any agency thereof, nor any of their bility for the accuracy, comply, express or implied, or assumes any legal liability or responsiprocess disclosed, or represents that its usefulness of any information, apparatus, product, or ence herein to any specific commercials use would not infringe privately owned rights. Refermanufacturer, or otherwise does not product, process, or service by trade name, trademark. mendation, or favoring by thes not necessarily constitute or imply its endorsement, recomand opinions of authors expressed States Government or any agency thereof. The views United States Government or any agency thereof.
} 


$$
\text { PuLSTH-19633 }
$$

\title{
THE USER ORIENTED EVALUATION PROCESS: A PROCESS FOR PRESERVING USER NEEDS DURING ITERATIVE SYSTEM TEST AND EVALUATION
}

\author{
Steven Hunt and Anne Schur \\ Pacific Northwest Laboratory \\ P.O. Box 999 \\ Richland, Washington 99352
}

\begin{abstract}
A system development process, called the User Oriented Evaluation (UOE) process, and an evaluation tool were created to place greater emphasis on user needs during computer system development. The UOE process is an iterative method for design that emphasizes the role of the user as the initiator of system requirements; evolutionary design requirement definition by enabling users and developers to experiment through the use of prototypes at all phases of system design; and appropriate utilization of developer and user areas of expertise. The evaluation tool is an integral part of the UOE process and provides the ability to solicit on-line meaningful feedback from users in real-time, and a means to capture a user's ongoing experience with the computer system. The paper contains a description of the UOE process and the evaluation tool, the capabilities of each and the history of their development.
\end{abstract}

\section{INTRODUCTION}

The ideal computer system is one that meets the user's needs and expectations. To create such a system, user needs must be the driving force behind system design. However, it is often difficult to identify user needs due to problems such as a complex user domain, limited interaction between users and developers, and difficulty in identifying user needs during test and evaluation. Often, users are only included in the design process as reviewers before the system is coded and during the test and evaluation which occurs toward the end of the implementation process. The users, when placed in this role, act as evaluators of a developer's system. This is opposed to making the users designers of a user centered system. The User Oriented Evaluation (UOE) process and its related evaluation tool, which is used to capture user comments during system test and evaluation, were created to assist developers in emphasizing user needs during system development.

Developers at Pacific Northwest Laboratory (PNL) were tasked to create a computerized research assistant for computational chemists (the Electronic Assistant for Scientific Investigation - EASI). Computational chemistry research requires the use of specialized complex computer applications to collect, integrate, synthesize and generate new information based on individual insight. It is difficult for a person not 
specifically trained in computational chemistry to truly comprehend this user domain. As a result, it was difficult for developers to initially identify user needs. Additionally, developer access to users was limited. Given these problems, it was necessary to establish a design process that would allow developers to create a usable system without becoming skilled in the user domain, and which would increase the benefits of any development efforts that involved users. The UOE process and the evaluation tool were the result of these efforts.

This paper is organized into the following five sections: Terminology, The UOE Process, The Evaluation Tool, Conclusions, and Future Work.

\section{TERMINOLOGY}

Table 1 gives a list of specific definitions that have been employed for terms used in this paper.

Table-1: Terms and Definitions

\section{THE USER ORIENTED EVALUATION PROCESS}

The UOE process is an iterative design method that emphasizes: the role of the user as the initiator of development; a clear definition of the roles of users and developers at all phases of system design; and evolutionary system development. Table 2 outlines the steps of the UOE process.

\section{Table-2: UOE Process Summary}

The UOE process begins with an initial meeting between users and developers in order to identify the overall capabilities that the users want the system to provide. This meeting should be in an environment where the developers and users can interact over a long period of time without interruptions. During this meeting the developers probe users for information and do not suggest possible solutions. The capabilities identified in this meeting provide a starting point for the UOE process described in Step 1.

Parallel prototype development is supported by the UOE process. For instance, several different prototypes could be created during Step 4, each addressing different levels of function detail. Each of these prototypes could result in discrete iterative cycles associated with development of different functions. The functions in each prototype could then be refined independently. Once they are completed, the functions are incorporated to create the final system.

Following are key aspects of the UOE process and the advantages they offer.

- Several prototypes may be developed simultaneously. By focusing the 
prototype design developers are able to concentrate on perfecting individual aspects of the system before incorporating them into the final system. Additionally, the users can more easily focus on specific functions during test and evaluation.

- System requirements are based on specific capabilities identified by the users. The developers do not make assumptions about which capabilities the users want in the system. Additionally, since functions development is determined by the capabilities, the users are able to initiate the creation of functions.

- The system design method is flexible, allowing the developers and users to experiment through the use of prototypes. The users are able to refine functions and introduce new capabilities throughout system development. Developers are able to try different functions in order to determine which ones best provide the required capabilities.

- Functions are tested by the users prior to system completion and function adequacy is determined by the users.

- The roles of the developers and users are clearly defined for each step of the process. Design decisions are made by individuals having the appropriate expertise. For example, capability definitions are not limited by user perceptions of system constraints, and developers do not make assumptions about which capabilities the users want.

- The system develops through compromises between the users' knowledge of desired capabilities and the developers' knowledge of system constraints. Functions that most closely meet the users needs can be created without requiring the developer to understand the user domain.

- Through use of the evaluation tool, user comments can be easily made and quickly incorporated into the system development. User input has an immediate impact on system development which contributes to user ownership of the system early in the system development life-cycle.

The UOE process is applicable throughout the life cycle of a project and at all levels of design, from very specific problems to large scale system development. It is designed to influence the way cievelopers and users think about system design at each step of the development process.

\section{THE EVALUATION TOOL}

The evaluation tool is an essential element of the UOE process. The tool, created at PNL during the development of EASI, is used to capture real-imie, cn-line comments from the user ciuring system testing (step 5 of the UOE process). It can be described as an electronic "Post-it" note that allows the user to make commonts within 
the system itself. The evaluation tool is placed in prototypes developed during the UOE process and is used extensively by the users to comment on current functions and express the need for additional capabilities.

The steps involved in using the evaluation tool.

1. The user identifies an aspect of the prototype to comment on.

2. The user selects the label "annotation" from the menu. This causes the cursor to change from an arrow to a cross hair.

3. By holding down the mouse button and moving the mouse the user is able to draw a rounded rectangle, the "annotation mark", on the screen. The user draws the annotation mark where they wish to make the comment. (Figure 1)

4. Once the area is marked the user releases the mouse button, which causes an annotation window to appear. The user then types the comment within this window. (Figure 2)

5. The user closes the annotation window and is returned to the prototype environment. The user can re-open the annotation window by placing the cursor in the annotation mark and pressing the mouse button.

Additional features provided by the evaluation tool are outlined in Table 3.

Table-3: Additional Evaluation Tool Features

This evaluation tool offers developers the following advantages:

- Comments about an aspect of the system can be made immediately when the aspect is encountered. As result, users are less likely to forget comments due to the time lapse between testing the system and reporting their impressions to the developers.

- Comments about system behavior can be made within the context of the system so the users don't have to describe where in the system the behavior occurred. This assists developers in understanding user comments and reduces the need for developers to be present during test and evaluation.

- Through users' comments, and lack of comments, the tool assists in the identification of functions that are not immediately clear to the users. This allows developers to determine which functions are immediately intuitive and which functions are learned through trial and error.

The evaluation tool provides developers with a powerful tool for capturing and analyzing user impressions during system test and evaluation.

\section{CONCLUSIONS}

Based on experience gained during the development of EASI, the UOE process 
and the evaluation tool greatly enhance the ability of developers to integrate user input into the software development life cycle. Because the UOE process clearly defines the role of users, it is possible to insure that focus is placed on users during system development. The evaluation tool allows this focus to be more fully exploited. The annotation tool and UOE process make it possible for the users to use developers as a tool for creating a system for their domain.

\section{FUTURE WORK}

Further development of the evaluation tool is in progress. This tool will include a usability monitor and usability workbench. The monitor will both explicitly (e.g. checklists for users to fill out) and implicitly (e.g. amount of time cursor resides over objects) measure performance parameters specified by system evaluators. The workbench will provide an environment for system implementers (e.g. computer scientists, human factors engineers) to specify parameters to be monitored, and will provide an environment for the analysis of the collected data. This effort should make available performance data as well as opinion based data. Also, it is planned to make the evaluation tool portable for use in prototype environments residing on other platforms such as the PC or the SUN.

\section{ACKNOWLEDGEMENTS}

This evaluation tool was developed using Silicon Beach Software's SuperCard and hosted on a Macintosh Ilfx, with a RasterOps 17 inch monochrome 1024 × 768 resolution monitor.

The creation of the Electronic Assistant for Scientific Investigation was internally funded by Pacific Northwest Laboratory. 


\begin{tabular}{|c|c|}
\hline TERMS & DEFINITIONS \\
\hline Capablilty & $\begin{array}{l}\text { An envistoned abllicy defined } \\
\text { by users that allows them to } \\
\text { achleve thier goal. }\end{array}$ \\
\hline Function & $\begin{array}{l}\text { Any aspect of a system that } \\
\text { provides users with a } \\
\text { capablllty. A function can } \\
\text { range from a complete system } \\
\text { to a single aspect of screen } \\
\text { design. }\end{array}$ \\
\hline Developer & $\begin{array}{l}\text { Any individual involved in the } \\
\text { system development process } \\
\text { who is not an end user. }\end{array}$ \\
\hline Intultive & $\begin{array}{l}\text { Describes any function the user } \\
\text { can utllize without prior formal } \\
\text { training or through trial and } \\
\text { error. }\end{array}$ \\
\hline Prototype & $\begin{array}{l}\text { A working model of proposed } \\
\text { runctlons. }\end{array}$ \\
\hline $\begin{array}{l}\text { Programmatic } \\
\text { Constraints }\end{array}$ & $\begin{array}{l}\text { Factors such as technological } \\
\text { leaslollity, cost, and scheduling } \\
\text { that can limit function } \\
\text { development. }\end{array}$ \\
\hline User & $\begin{array}{l}\text { Any individual who works in the } \\
\text { domain for which the system is } \\
\text { being bullt and who will use the } \\
\text { completed system. }\end{array}$ \\
\hline
\end{tabular}




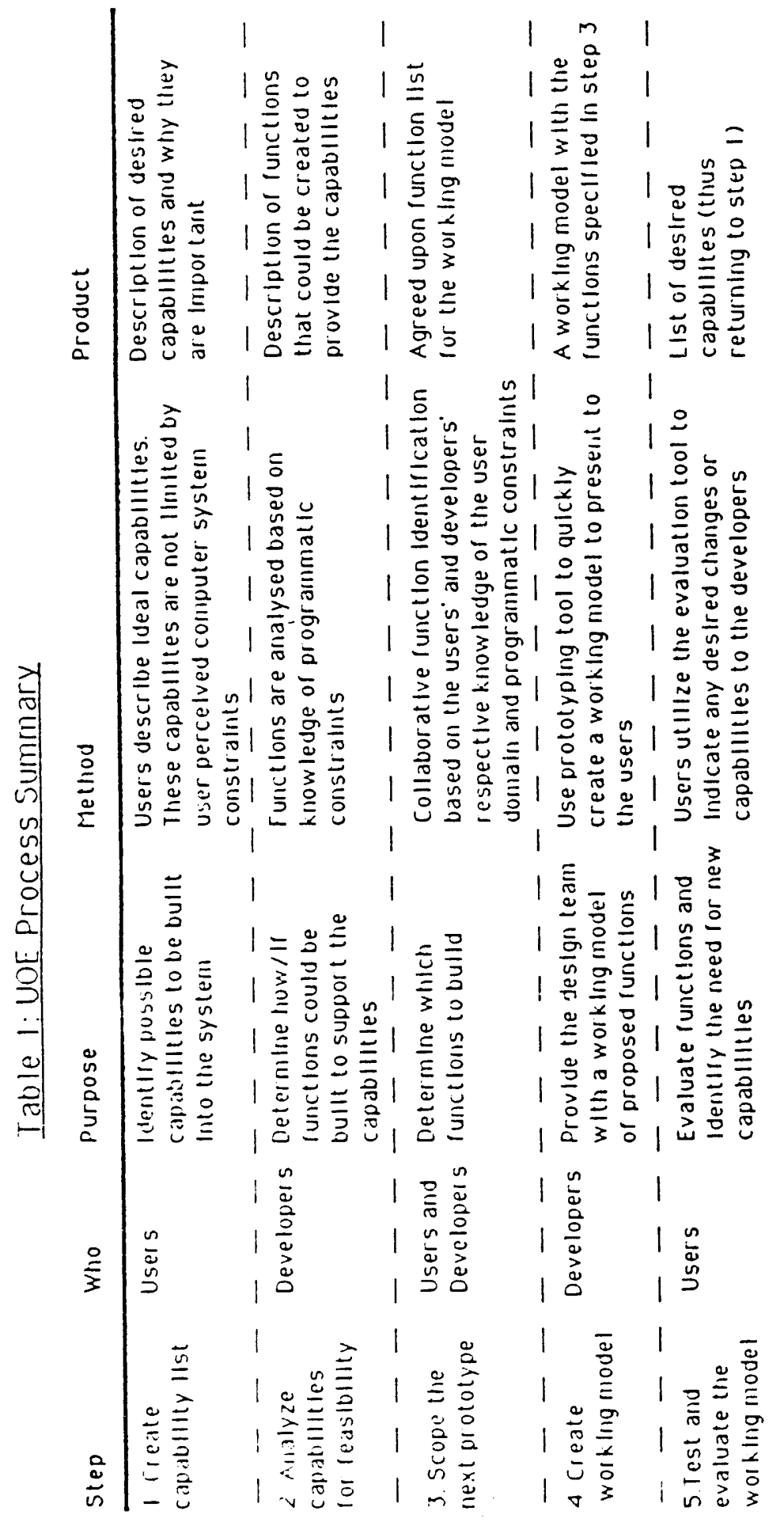


Annotation marks can be placed anywhere within the prototype system, even inside annotation windows.

When created, annotations are automatically labeled with the user name, date and time.

Annotation comments can also be titled.

Annotation comments can be accessed from within the system at any time by placing the cursor on the annotation mark and pressing the mouse button.

All annotation comments can be placed in a separate file and accessed out of context of the system.

Annotations can be sorted and searched by title, date or keywords.

All annotation marks and comments are automatically saved when the user either closes the annotation window or leaves the prototype.

The ability to insert, delete and move text is provided in the comment area of the annotation window.

Annotation marks and windows can be moved at any time.

$$
\text { Toll }
$$




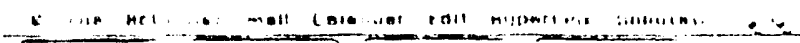

$\sin 1+2+1$

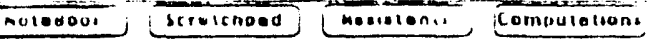

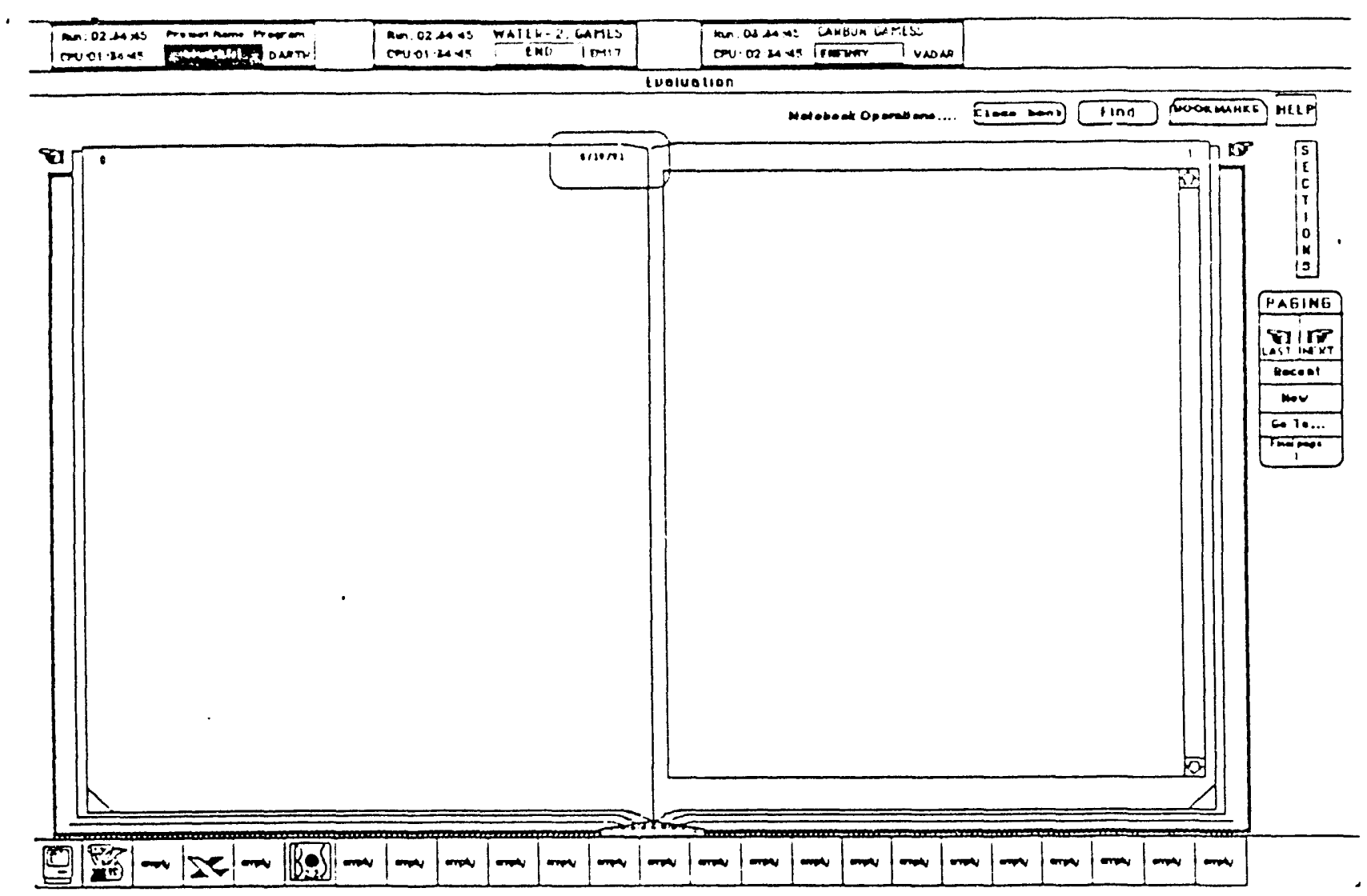




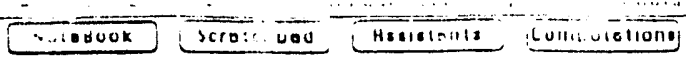

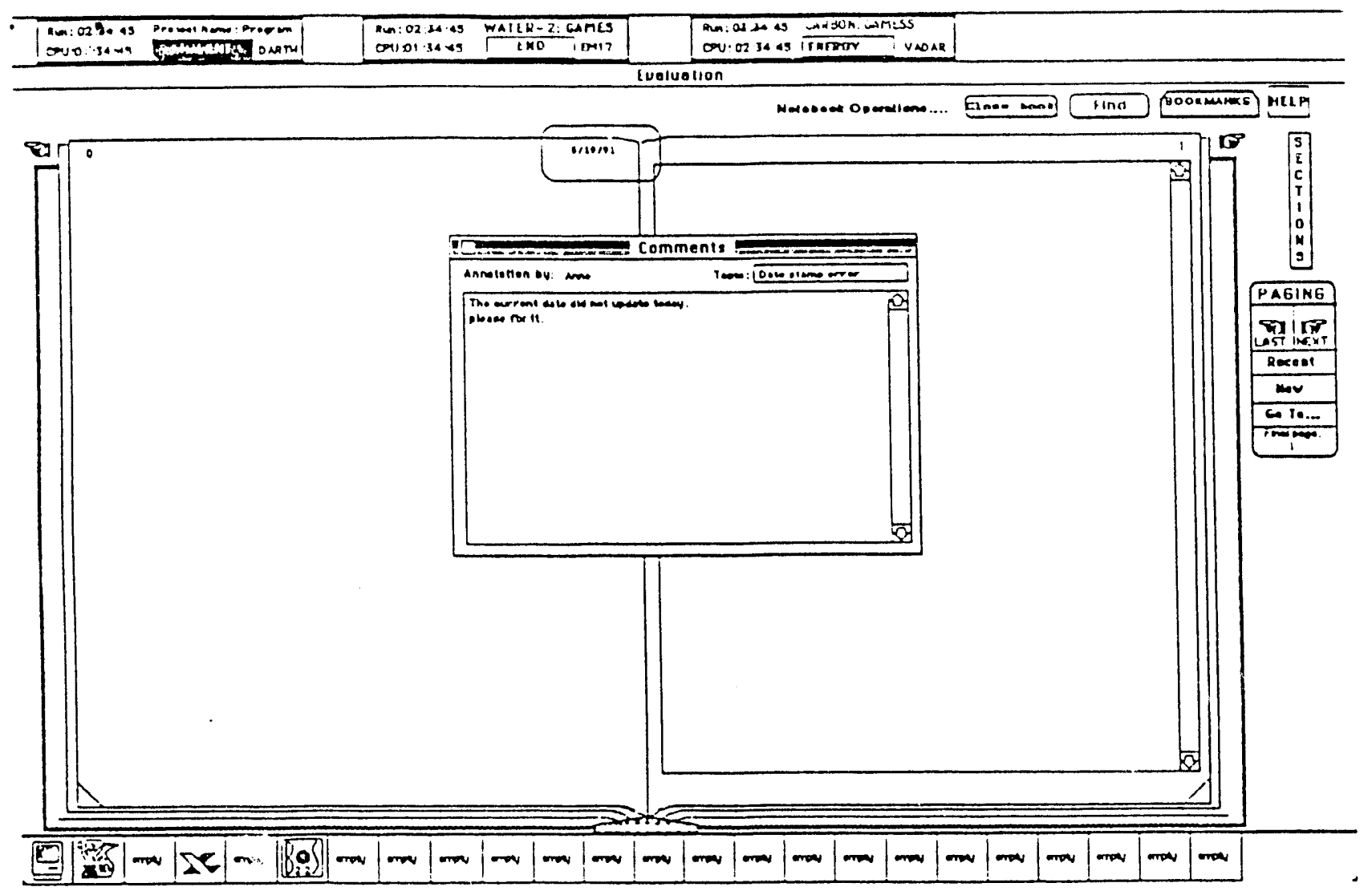



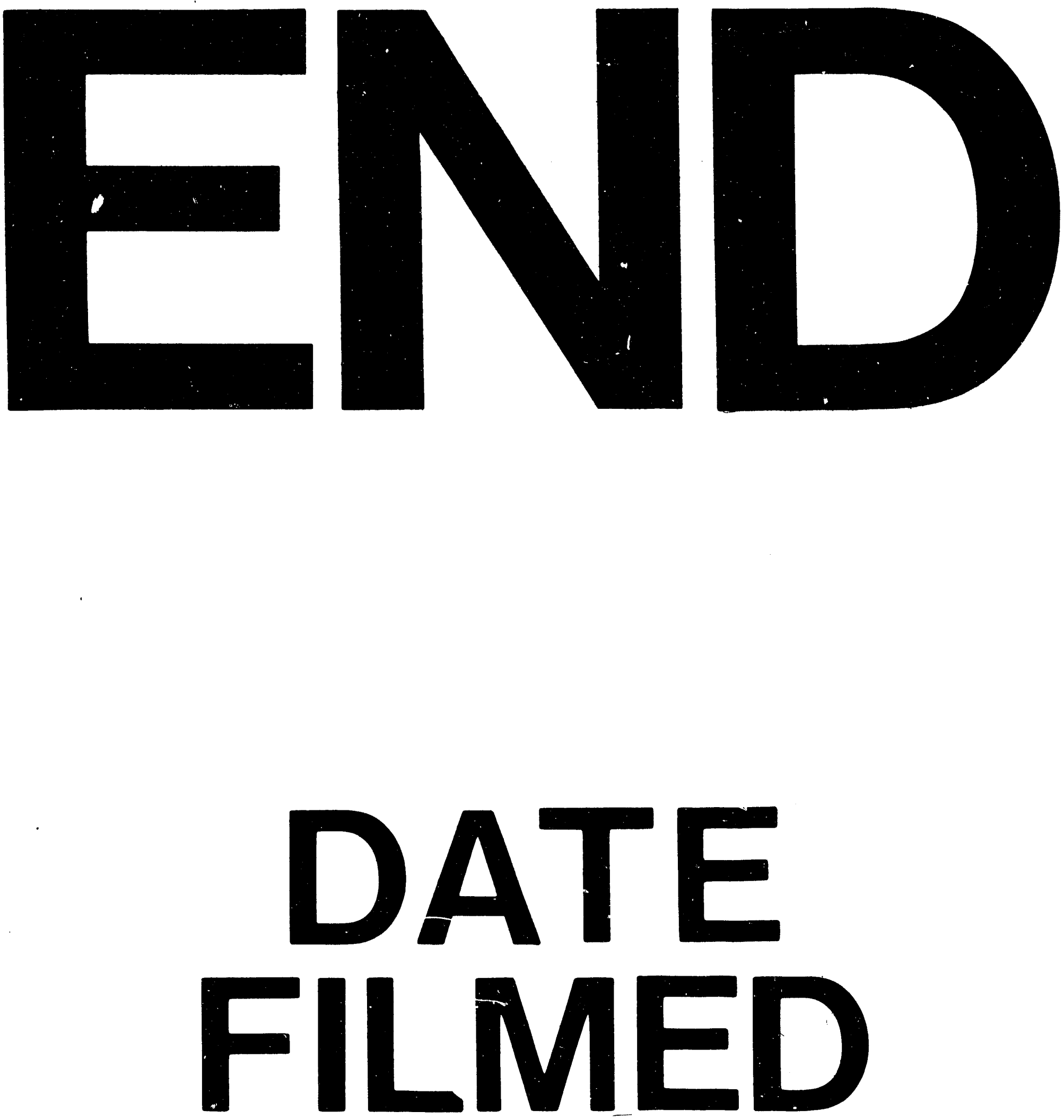

1

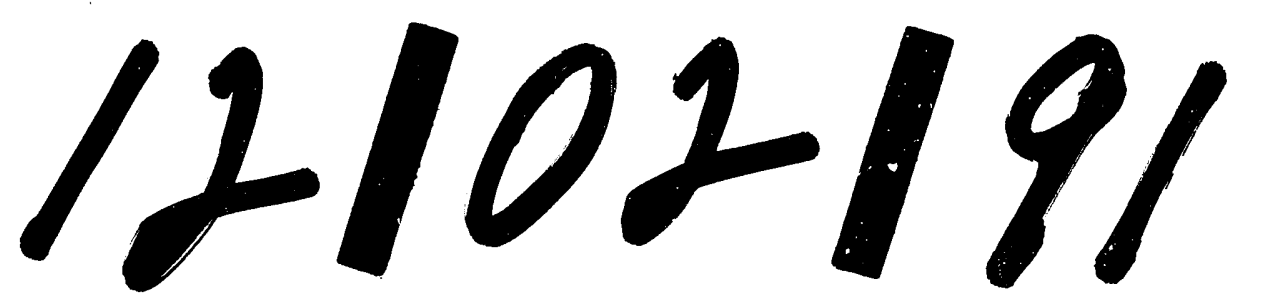

